\title{
Determination of the coefficients of Langevin models for inhomogeneous turbulent flows by three-dimensional particle tracking velocimetry and direct numerical simulation
}

\section{Citation for published version (APA):}

Walpot, R. J. E., Geld, van der, C. W. M., \& Kuerten, J. G. M. (2007). Determination of the coefficients of Langevin models for inhomogeneous turbulent flows by three-dimensional particle tracking velocimetry and direct numerical simulation. Physics of Fluids, 19(4), 045102-1/13. [045102]. https://doi.org/10.1063/1.2717688

DOI:

10.1063/1.2717688

Document status and date:

Published: 01/01/2007

\section{Document Version:}

Publisher's PDF, also known as Version of Record (includes final page, issue and volume numbers)

\section{Please check the document version of this publication:}

- A submitted manuscript is the version of the article upon submission and before peer-review. There can be important differences between the submitted version and the official published version of record. People interested in the research are advised to contact the author for the final version of the publication, or visit the $\mathrm{DOI}$ to the publisher's website.

- The final author version and the galley proof are versions of the publication after peer review.

- The final published version features the final layout of the paper including the volume, issue and page numbers.

Link to publication

\footnotetext{
General rights

- You may freely distribute the URL identifying the publication in the public portal. follow below link for the End User Agreement:

www.tue.nl/taverne

Take down policy

If you believe that this document breaches copyright please contact us at:

openaccess@tue.nl

providing details and we will investigate your claim.
}

Copyright and moral rights for the publications made accessible in the public portal are retained by the authors and/or other copyright owners and it is a condition of accessing publications that users recognise and abide by the legal requirements associated with these rights.

- Users may download and print one copy of any publication from the public portal for the purpose of private study or research.

- You may not further distribute the material or use it for any profit-making activity or commercial gain

If the publication is distributed under the terms of Article $25 \mathrm{fa}$ of the Dutch Copyright Act, indicated by the "Taverne" license above, please 


\title{
Determination of the coefficients of Langevin models for inhomogeneous turbulent flows by three-dimensional particle tracking velocimetry and direct numerical simulation
}

\author{
R. J. E. Walpot, C. W. M. van der Geld, and J. G. M. Kuerten ${ }^{\text {a) }}$ \\ Department of Mechanical Engineering, Eindhoven University of Technology, P.O. Box 513, \\ $5600 \mathrm{MB}$ Eindhoven, The Netherlands
}

(Received 24 August 2006; accepted 25 January 2007; published online 13 April 2007)

\begin{abstract}
A promising and, in terms of computer power, low-cost way of describing flow properties such as turbulent diffusion is by Langevin models. The development of such models requires knowledge of Lagrangian statistics of turbulent flows. Our aim is to determine Lagrangian statistics of inhomogeneous flows, as most turbulent flows found in practical applications are inhomogeneous. The present paper describes how a Lagrangian measurement technique, three-dimensional particle tracking velocimetry, has been developed and applied to the most common example of inhomogeneous flows: turbulent pipe flow. A new direct numerical simulation (DNS) code has been developed and experimental results have been compared with results of this DNS code. The results concern Eulerian and Lagrangian velocity statistics at two Reynolds numbers. Based on these, coefficients of the Langevin model have been determined and physical consequences for Langevin modeling and turbulent dispersion have been explained. (C) 2007 American Institute of Physics. [DOI: $10.1063 / 1.2717688]$
\end{abstract}

\section{INTRODUCTION}

Stochastic models are a useful way to describe particle dispersion in turbulent flows. For the development of such models, knowledge of Lagrangian statistics of turbulent flows is useful, for example to calculate an unknown damping function included in such a model. Taylor's $1921^{1}$ paper on turbulent dispersion describes the Langevin equation for stationary, homogeneous, isotropic turbulence, in which case the damping coefficient is a constant. Sawford's 2001 review article $^{2}$ gives an overview of the history and development of Lagrangian models since then. A stochastic Lagrangian model for particle velocity in homogeneous shear flow was developed by Pope. ${ }^{3}$ For many other kinds of turbulence, as occurring in common practice, a unique formulation for the damping function has yet to be found. Rather than focusing on homogeneous turbulent flows, the present research aims at measurement of inhomogeneous turbulent flow in a pipe, to enable determination of the damping function.

The need for Lagrangian statistics makes experimental methods that apply to a Lagrangian frame of reference indispensable. Such techniques have rapidly been developed over the past few years and presently several fully threedimensional Lagrangian techniques are available. They can be divided into two main categories, namely optical and sonar techniques. The main advantage of the sonar techniques ${ }^{4}$ is the direct measurement of particle velocities using Doppler shifts. Another advantage is that measurements in nontransparent media are possible at high sampling frequencies, up to a few $\mathrm{kHz}$. The drawbacks of this technique are related to the seeding of the fluid with tracer particles. The working principle and software algorithms applied currently demand

$\overline{{ }^{a} \text { Electronic mail: j.g.m.kuerten@tue.nl }}$ a relatively large seeding size, in the order of tenths of millimeters, and very low seeding densities, in most cases not more than one particle in the measurement volume at a certain time. The large size and thus the far from ideal flow tracing characteristics limit this technique to low flow velocities, and the low seeding density demand is not favorable when gathering statistical averages of flow quantities. A sonar technique has been used by Mordant et al. ${ }^{5}$ to determine long-time Lagrangian velocity correlations, in order to study intermittency in turbulence. Seeding densities and sizes are more flexible in the case of optical techniques such as threedimensional particle tracking velocimetry (3D PTV). ${ }^{6-14}$ Virant and Dracos studied the boundary layer of free-surface turbulent flow using 3D PTV. ${ }^{7}$ The turbulent diffusion of particle pairs in oscillating grid turbulent flow was studied by Ott and Mann. ${ }^{11}$ Suzuki and Kasagi ${ }^{13}$ used the 3D PTV technique, originally developed by Nishino et al. ${ }^{12}$ for liquid flows, to study secondary flow effects in turbulent air flow through a curved square bend. In other 3D PTV researches, a fully developed quasihomogeneous turbulent flow of the von Kármán swirling type was studied. ${ }^{6,10,14}$ Voth et al. ${ }^{10}$ measured particle accelerations in this flow type, using highspeed silicon strip detectors, in order to determine universal characteristics of turbulent flows. ${ }^{10,14}$ Lüthi et al. ${ }^{6}$ measured the full set of velocity derivatives, to study the role of viscosity on the enstrophy budget.

As indicated by Hoyer et al.,${ }^{15}$ the maximum number of particles that can be tracked simultaneously by 3D PTV systems gradually increased to $\mathcal{O}\left(10^{3}\right)$ since the introduction of the technique in the 1980s. This number is thought to represent the limit for classical 3D PTV systems with four cameras. Traditional 3D PTV algorithms can be divided into two parts: determination of the particle positions in space and the 
tracking of the particles in time. Willneff ${ }^{8}$ combined these two steps and the resulting spatio-temporal matching algorithm improves particle tracking efficiency by $10-30 \%$. This makes it unnecessary to lower seeding density in order to increase particle matching probability. For the same reason, a new technique called 3D scanning PTV is developed by Hoyer et al. ${ }^{15}$ Changes in illumination, image acquisition, and analysis lead to a significant increase in the maximum number of trackable particles, which is especially useful when spatial derivatives of particle velocity are of interest.

In our case, the goal is to accurately determine Lagrangian statistics, such as velocity correlation functions. This demands that a large number of fluid or tracer particles can be followed over a significant time interval and the camera sampling frequency is required to be at least twice the characteristic frequency of the flow phenomena of interest. At low or moderate Reynolds numbers, commercially available highspeed cameras with sampling frequencies of $30 \mathrm{~Hz}$ or more are usually sufficient. It can therefore be concluded that 3D PTV is a suitable method for the determination of Lagrangian statistics as long as the frequency of the image recording equipment is sufficient to capture all relevant flow phenomena, whereas sonar techniques and the fast image detectors suffer from low seeding densities and short measurement times, respectively.

The present paper describes how velocity statistics of turbulent pipe flow and its consequences for Langevin modeling have been determined using 3D PTV and DNS. A description of the experimental and data processing techniques is given in Sec. II. To our knowledge, the application of a Lagrangian experimental technique to pipe flow has not been done before. Results will be compared with direct numerical simulation (DNS) results in Secs. III and IV. Accurate DNS predictions of turbulent pipe flow are scarce, and a description of a new DNS code as developed by Veenman ${ }^{16}$ (referred to as VKW in the remaining part of this paper) is given in Sec. II D. With our measuring equipment, it is at present still impossible to measure at Reynolds numbers higher than the ones at which DNS results can be obtained. Comparison with DNS will prove that Lagrangian experimental techniques are applicable to the research of inhomogeneous turbulent flows. This is done for turbulent water flow through a pipe at two Reynolds numbers. Based on the bulk velocity and inner pipe diameter, these Reynolds numbers are $\operatorname{Re}_{b}=U_{b} D / \nu=5300$ and $\operatorname{Re}_{b}=10300$, with $\nu$ the kinematic viscosity, which is equal to $\nu=1 \times 10^{-6} \mathrm{~m}^{2} \mathrm{~s}^{-1}$ for water at atmospheric conditions. Lagrangian results will be compared with results of the VKW DNS in Sec. IV. In the same section, the Kolmogorov constant and damping function, to be specified below, will be determined from Lagrangian statistics. Physical implications for the development of the Langevin model are discussed. The same section describes the Reynolds limit for the current hardware, and possibilities to increase this limit. As Lagrangian statistics of turbulent pipe flow are almost absent in the literature, Eulerian velocity statistics are also studied for validation purposes in Sec. III. Finally, conclusions are presented in Sec. V.

\section{THEORETICAL BACKGROUND AND EXPERIMENTAL TECHNIQUE}

\section{A. Theoretical background}

As mentioned in the Introduction, a Lagrangian stochastic model is often an efficient way of predicting, for example, turbulent dispersion. An example of such a model is the Langevin equation for fluid particle velocity (here, fluid particles are passive particles that have no mass and that move with the local fluid velocity),

$$
\frac{d u_{i}^{\prime}(t)}{d t}=A_{i j} u_{j}^{\prime}(t)+\left(C_{0} \epsilon\right)^{1 / 2} w_{j}(t),
$$

$u_{i}$ denotes one of the three velocity components in a Cartesian frame of reference. The usual distinction between the mean $\langle u\rangle$ and fluctuating part $u^{\prime}$ of the total velocity $u=\langle u\rangle$ $+u^{\prime}$ is made, where the brackets signify ensemble averaging. $A_{i j}$ is called the drift tensor or damping tensor, $C_{0}$ is the Kolmogorov constant, $\epsilon$ is the energy dissipation rate, and $w_{j}(t)$ is random white noise. Veenman ${ }^{16}$ shows that the unknown damping tensor can be determined from nonnormalized Lagrangian velocity correlation functions,

$$
\rho_{i j}(\tau, r)=\left\langle u_{i}^{\prime}\left(t_{0}\right) u_{j}^{\prime}\left(t_{0}+\tau\right)\right\rangle,
$$

where $t_{0}$ denotes the reference time and $\tau$ the time separation. The correlation functions depend on the radial coordinate but are independent of $t_{0}$. Equation (2) therefore only yields correct results if all tracer particles considered start with the local fluid velocity at the same radial coordinate $r$ at time $t_{0}$. The particles thus need to be delta-distributed in the radial direction at time $t_{0}$. This situation can easily be created in numerical simulations but is impossible in experiments. The calculation of correlation $\rho_{i j}(\tau, r)$ is thus done by averaging over all particles that are situated at radial position $r$ at a certain time, which is then marked $t_{0}$ for that particle. The particles are selected using the sampling method described in Sec. II C. The 3D PTV and DNS results are used to calculate the coefficients of the damping tensor and the Kolmogorov constant in Secs. IV A and IV B, respectively.

In the remaining part of this section, the most important features of the experimental technique are described. The 3D PTV method and setup are discussed in detail in Sec. II B. Acquiring statistics from raw 3D PTV data requires special attention, as is described in Sec. II C. Information on the numerical technique of the new VKW DNS is included in Sec. II D.

\section{B. 3D PTV method}

The 3D PTV setup consists of a camera frame for reliable and accurate positioning of the cameras, and a calibration unit to determine the relation between camera and realworld coordinates. By removing a tube section, the calibration unit can be placed on top of the measurement section, as shown in Fig. 1. This enables in situ calibration by moving a well-defined calibration grid through the measurement volume. The grid is manufactured out of a glass plate that is coated on one side. The grid points are made out of circular voids in the coating. To facilitate recognition of 


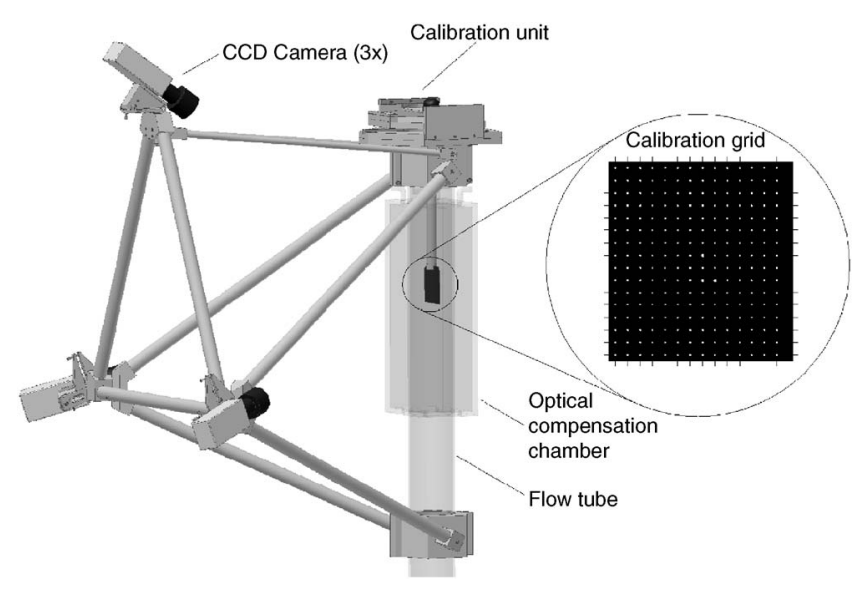

FIG. 1. The 3D PTV setup with the calibration unit in place.

the $X$ and $Y$ directions, three larger dots are positioned in the center of the grid.

Because of the statically determined design of the camera frame, which is described in detail in a previous publication, ${ }^{17}$ there is no incorporation of unknown stresses in the frame or flow tube. The cameras are attached to the flow tube instead of mounting them on separate camera support stands. Relative motion of the cameras to the measurement volume is avoided in this way.

To create a stable and accurately controlled turbulent flow, a special water circulation system has been built. The measurement section consists of a vertically oriented glass pipe, with an inner diameter, $D$, of $100 \mathrm{~mm}$ and a length of $70 D$, to ensure optical accessibility. A water-filled rectangular glass box surrounds the measurement volume to minimize optical distortions. To facilitate temperature stabilization, a water reservoir with a capacity of $2 \mathrm{~m}^{3}$ is included in the system. A frequency controlled pump forces the water through the pipe and a flow straightener, a tube bundle conditioner installed in the lowest part of the test section according to ISO standard ${ }^{18} 5167-1: 1991$ to discard all secondary flow effects. The system is described in more detail in a previous publication. ${ }^{17}$

To visualize the flow, polyamide seeding particles with diameters between 200 and $250 \mu \mathrm{m}$ are added to the water flow. The particles have a mass density close to that of water under atmospheric conditions $\left(\rho_{p}=1030 \mathrm{~kg} / \mathrm{m}^{3}\right)$ and are close to being spherical. Of course, these particles have to be able to follow all velocity fluctuations of the flow. The most rapid fluctuations take place on time scales on the order of the Kolmogorov time scale, for which an order-of-magnitude estimation can be made by using $\tau_{k}=(\nu / \epsilon)^{1 / 2}$, where $\nu$ is the kinematic viscosity and $\epsilon$ is the kinetic energy dissipation per unit mass. For water flow under atmospheric conditions, $\nu$ $\approx 10^{-6} \mathrm{~m}^{2} \mathrm{~s}^{-1}$ and the energy dissipation can be expressed as $\epsilon=4 u_{\tau}^{2} U_{b} / D$; see Ref. 19 , where $u_{\tau}$ is the wall shear velocity and $D$ is the pipe diameter. In the present paper, we focus on moderate Reynolds numbers, for example $\mathrm{Re}_{b}=5300$, in which case $\tau_{k} \approx 0.29 \mathrm{~s}$. The relaxation time for small particles in stationary flow is shown by Albrecht et al. ${ }^{20}$ to be

$$
\tau_{p}=\left(d^{2} \rho_{p} / 18 \mu\right)\left(1+0.5 \rho_{f} / \rho_{p}\right),
$$

where $d$ is the particle diameter, $\rho$ is the mass density, and $\mu$ is the dynamic viscosity. The subscripts $p$ and $f$ stand for particle and fluid, respectively. For the earlier mentioned $250 \mu \mathrm{m}$ seeding particles, we obtain a relaxation time of $\tau_{p} \approx 5 \mathrm{~ms}$. As $\tau_{p} \ll \tau_{k}$, it can safely be assumed that the particles follow all flow fluctuations.

To optimize the accuracy of determination of the particle position, the optical setup is such that the tracer particles appear on the camera CCD with a diameter of a few pixels. This facilitates the use of a subpixel accurate gray-value weighted average determination of tracer particle center. For particles with a typical diameter of about 10 pixels, as in our experiments, the error in the determined center is then less than 0.1 pixel. $^{21}$

The illumination of the measurement volume is realized by two strong stroboscopic light sources of about $5 \mathrm{~J}$ light energy per pulse each. The strobes are custom-made in-house for this project to optimize light output and camera synchronization; they have a maximum pulse frequency of about $100 \mathrm{~Hz}$ and can be synchronized to the cameras with a negligible delay. The light pulse duration is approximately $40 \mu \mathrm{s}$, ensuring virtually instantaneous seeding particle images for the Reynolds numbers considered. At $\operatorname{Re}_{b}=5300$, a seeding particle traveling at the maximum fluid velocity undergoes a displacement of about $3 \mu \mathrm{m}$ during the light pulse, which is much smaller than its diameter. The stroboscopes enable illumination of the entire measurement volume of about $85 \times 120 \times 50 \mathrm{~mm}^{3}$ (width $\times$ height $\times$ depth) without any significant heat generation.

The principle of 3D PTV requires a minimum of two synchronized cameras to capture the instantaneous 3D particle positions in the measurement volume, but the use of more cameras minimizes ambiguities in particle detection and allows for higher seeding densities. As we are interested in Lagrangian velocity statistics, and not in spatial derivatives, a high seeding density is not a primary requirement, and the use of a three-camera system is sufficient. The cameras (Kodak MegaPlus ES1.0) have a 10-bit grayscale CCD with a resolution of $1018 \times 1008$ pixels and a maximum sampling frequency of $30 \mathrm{~Hz}$. The 3D particle tracks are determined using the 3D PTV algorithm developed by Kieft et al. ${ }^{9,22}$ who based their method on the work of Yamamoto et $a .^{23}$ The algorithm starts with a filtering technique called dynamic thresholding to get rid of background noise in the obtained images. Subsequently, the 2D representation of a particle is detected within each camera image and, combined with the camera calibration information, the 3D particle position is determined. This procedure is schematically depicted in Fig. 2, where dynamically thresholded images of all three cameras are shown alongside typical 3D particle position information at a single time step. Finally, the algorithm checks which particle in frame $i+1$ is most likely to match to a particle in frame $i$. During this last step, information of previous matches of the current particle and neighboring particles, up to frame $i$, is used to extrapolate the particle track to the most likely position in frame $i+1$. This algorithm does not contain the improvements to classical 3D PTV intro- 

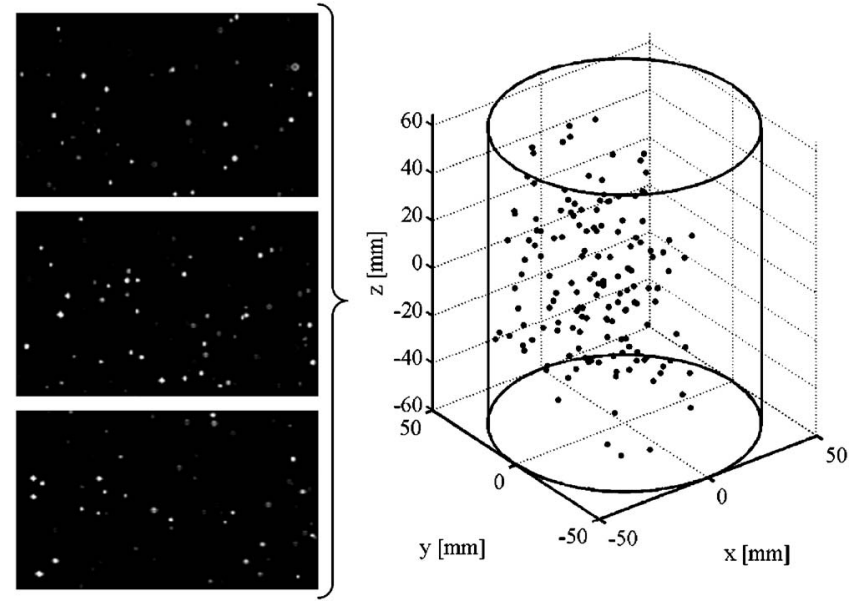

FIG. 2. Samples of dynamically thresholded images from each of the three cameras, with on the right-hand side a typical example of recognized 3D particle positions at a single time step.

duced by Refs. 8 and 15, as mentioned in the Introduction. Nevertheless, it shows good performance in tracking the relatively simple particle motion encountered in pipe flow, and in the test case published by Kieft et al., ${ }^{9}$ who studied the wake flow behind a heated cylinder.

\section{Data processing}

In pipe flow, inhomogeneous behavior is encountered in the radial direction. This radial dependency necessitates selected sampling of the measured particle tracks, in contrast to homogeneous, isotropic turbulence, where Lagrangian statistics can be determined by ensemble averaging over particles that are arbitrarily distributed over the entire domain. The easiest way to perform the sampling in pipe flow is to take only those particles into account that cross a certain radial position, $r_{\text {crit }}$. This would, however, result in preferential sampling of particles having a high radial velocity because they are more likely to cross a chosen radial position. This problem is solved by using the particle sampling method presented by Walpot et al. ${ }^{24}$ If the instantaneous particle position $r$ satisfies

$$
r_{\text {crit }}-\Delta r<r<r_{\text {crit }}+\Delta r,
$$

then $r$ is considered as corresponding to a useful particle trajectory. Here $\Delta r$ denotes half the width of the sampling volume. All particles that can be found inside the specified volume at a certain time are taken into account. At each time step that a particle is situated within the specified volume, a ghost particle is created. Because particles with a low radial velocity are less likely to move to other radial positions, they will create more ghost particles than faster ones. All ghost particles contribute to the statistical averages, thus compensating for the velocity bias; see Ref. 24. A disadvantage of this method is that a particle with a relatively high radial velocity can pass $r_{\text {crit }}$ while it is never detected within the specified volume at a discrete time step. We found that the requirement $2 \Delta r>|u| \Delta t$, with $u$ a typical large radial velocity value, is appropriate to prevent this.
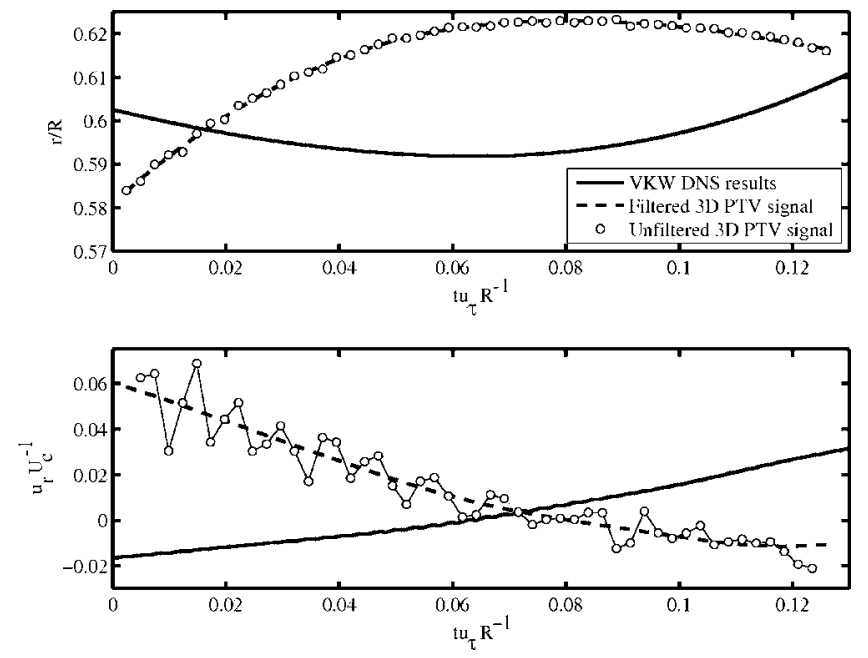

FIG. 3. Typical 3D PTV position and corresponding velocity signal before and after the application of the Savitzky Golay smoothing filter, represented by circles and dashed lines, respectively. A typical DNS particle trajectory and the corresponding velocity signal are plotted with solid lines.

The 3D PTV algorithm employed calculates 3D particle tracks according to the procedure as described in Sec. II B. High-frequency measurement noise is removed with a lowpass smoothing filter as was first introduced by Savitzky and Golay. ${ }^{25}$ The most rapid, physical velocity fluctuations are expected to have a characteristic frequency close to the Kolmogorov frequency, $1 / \tau_{k}$. To obtain a more accurate estimation of the Kolmogorov time scale as in Sec. II B, it has been estimated using spectral densities as calculated from DNS. The largest meaningful frequency in the spectrum appeared to be about $1 \mathrm{~Hz}$ for $\mathrm{Re}_{b}=5300$ and about $2 \mathrm{~Hz}$ for $\mathrm{Re}_{b}$ $=10300$. The measurement noise, which induces highfrequency disturbances on the position signal, is expected to have a big influence on the unfiltered autocorrelation function for small time separations. The unfiltered 3D PTV position signal contains sudden position jumps due to optical disturbances and the finite spatial resolution of the cameras. These position jumps result in large, nonphysical, velocity fluctuations causing an artificial increase of the velocity RMS and thus an increase of the starting value of the correlation function, because $\rho_{i j}(0)=\sigma_{i j}^{2}$. The high-frequency measurement noise was indeed found to cause the superposition of noise on the correlation functions calculated from unfiltered 3D PTV results. To obtain a suitable cutoff frequency, a filter span of 17 points with order 3 is used. The filter process is repeated 10 times to improve the stop-band attenuation. The transition bandwidth of the filter has of course a finite width. The above settings are found to work well for both Reynolds numbers, as will be shown later. To illustrate the necessity of the smoothing filter, Fig. 3 shows a typical 3D PTV particle position and corresponding velocity signal before and after the application of the smoothing filter. For comparison of typical fluctuation time scales, VKW DNS results are also included in the figure and represented by the solid lines.

In the VKW DNS computations, the particle velocity is interpolated to intergrid point particle positions using a 


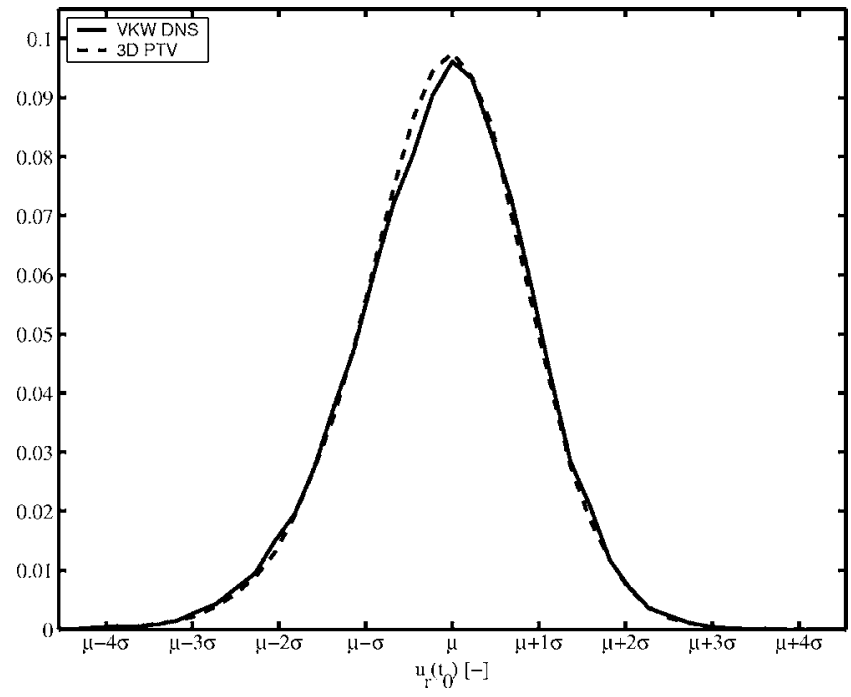

FIG. 4. Radial velocity PDFs for DNS results and filtered 3D PTV data.

fourth-order accurate interpolation scheme. This Lagrange interpolation scheme causes oscillations in the velocity signal, as is visible in Fig. 3 and also in the first part of some correlation functions, discussed in Sec. IV A. This approach was compared by Veenman ${ }^{16}$ with a direct summation of the Fourier series in the periodic directions and Hermite interpolation in the radial direction, which gives smooth velocity signals. We found that the oscillations have little or no influence on the low-order statistics in which we are interested, such as correlation functions. More details on the numerical method used in the VKW DNS are given in Sec. II D.

Figure 4 shows an example of radial velocity probability density functions (PDFs) as obtained by DNS computations and 3D PTV measurements at $r / R=0.5$ for $\mathrm{Re}_{b}=5300$. The solid line represents VKW DNS results whereas the 3D PTV results are denoted by the dashed line. Figure 4 shows that the full radial velocity PDF is captured well within $\pm 5 \sigma$ from the mean $\mu$, which also holds for other radial positions and velocity components. This justifies the exclusion of particle matches with more than $\mu \pm 5 \sigma$ fluctuations from 3D PTV data analysis. It has been found that the PDFs do not change if this exclusion is shifted to $\mu \pm 10 \sigma$, which implies that the method is not sensitive to outliers.

\section{DNS method}

In the numerical method for the VKW DNS, a finite part of a cylindrical pipe of length $5 D$ with periodic boundary conditions in the streamwise direction is used. Since both the streamwise and the tangential directions are periodic, the use of a spectral method is convenient. In the two periodic directions, a Fourier-Galerkin method is applied, and in the radial direction a Chebyshev-collocation method is applied. In order to avoid a large number of collocation points near the axis, the radial direction is first divided in several elements and in each element a Chebyshev expansion is chosen with continuously differentiable solutions at the element boundaries. In this way, it is also possible to reduce the number of tangential Fourier modes in the element closest to the axis. In total, 106 collocation points are taken in the radial direction and 128 Fourier modes in both periodic directions.

The vorticity formulation of the Navier-Stokes equation in cylindrical coordinates is discretized. The nonlinear terms in the Navier-Stokes equation are computed in physical space; FFT is applied to transform the solution from Fourier space to physical space and back. The $\frac{3}{2}$ rule prevents aliasing. The pressure and viscous terms are easily calculated in Fourier space. For time integration, a second-order time splitting is applied: an explicit second-order AdamsBashforth method for the nonlinear terms and an implicit method for the linear terms. The boundary condition for the pressure on the wall of the pipe is chosen in such a way that the velocity field is, in good approximation, divergence-free.

In order to determine the Lagrangian statistics, passive particles that move with the local instantaneous fluid velocity are tracked. In order to integrate the equations of motion of the particles in time, the first-order Euler forward method is used, but it has been checked that this is sufficiently accurate by comparing results with those of a second-order accurate Runge-Kutta method. The fluid velocity at the position of a particle is found from fourth-order accurate interpolation: Lagrange interpolation in the two periodic directions, and Hermite interpolation in the radial direction.

\section{EULERIAN STATISTICS}

In this section, Eulerian turbulent pipe flow statistics as determined with 3D PTV are presented and compared with various DNS results from the literature. Section IV will show Lagrangian velocity correlation functions as determined using 3D PTV and DNS. Lagrangian as well as Eulerian results are made available from the VKW DNS code. Other Lagrangian statistics for inhomogeneous flows have not been found in the literature, but Eulerian results could also be verified by DNS results as published by Eggels et al. ${ }^{26}$ (EGG), Loulou ${ }^{27}$ (LLO), and Wagner et al. ${ }^{28}$ (WAG). In the graphs below, error bars are plotted to indicate the magnitude of the error in the mean of a certain quantity for the experimental results. The size of the error bars is taken to be equal to $\pm 2 \sigma_{m}$, where

$$
\sigma_{m}=\sqrt{\left[\sum x_{i}^{2}-\frac{1}{n}\left(\sum x_{i}\right)^{2}\right] /[n(n-1)]}
$$

with $x_{i}$ the average value for a single set and $n$ the number of measurement sets. The probability that the actual mean value lies within the given error bars is about $95 \%$, see Squires. ${ }^{29}$ Whenever appropriate, velocity statistics are normalized by the centerline velocity, $U_{c}$, and plotted against the dimensionless distance to the pipe centerline, $r / R$. The centerline velocity is chosen as a normalization quantity instead of the wall shear velocity, $u_{\tau}$, that is often used in the literature, because $U_{c}$ can be determined more accurately in an experimental setup. Laser Doppler anemometry (LDA) velocity measurements have been performed simultaneously with the 3D PTV measurements to check the centerline velocity. The LDA time records also show that the velocity signal is random but stationary in time. 


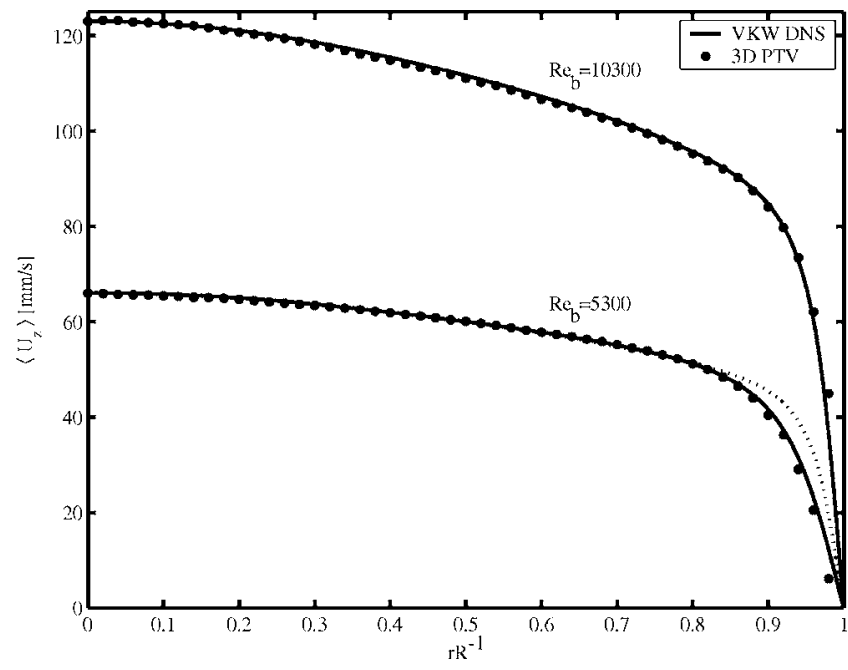

FIG. 5. Mean axial velocity profile for two Reynolds numbers. The solid lines represent the overlapping VKW and EGG DNS results for $\operatorname{Re}_{b}$ $=5300$ and $\mathrm{VKW}$ and $\mathrm{WAG}$ for $\mathrm{Re}_{b}=10300$. The dotted line represents the $\operatorname{Re}_{b}=10300$ data rescaled to match the $U_{c}$ of $\operatorname{Re}_{b}=5300$.

The 3D PTV results for $\mathrm{Re}_{b} \approx 5300$ are averaged over 50 separate measurement sets of 2 min each. The measurements were performed with a frequency of $30 \mathrm{~Hz}$, and at each time step, on average, 150 particles were recognized. The seeding density was deliberately kept this low to maximize the length of the measured particle tracks. The average Reynolds number during the 50 sets was $\operatorname{Re}_{b}=5320 \pm 30$. Before the start of each measurement set, the temperature and bulk velocity of the water was measured. The frequency controller of the pump facilitates accurate control over the velocity and thus, for constant fluid temperature, the Reynolds number. The average Reynolds number for the $\mathrm{Re}_{b} \approx 10300$ 3D PTV data is $\mathrm{Re}_{b}=10290 \pm 40$. For this Reynolds number, 40 measurement sets of 2 min each are available, with an average number of 100 recognized particles per time step.

The VKW DNS results are ensemble averaged over 600 velocity fields, covering 850 dimensionless time units where time is made dimensionless using the bulk velocity $U_{b}$ and the pipe diameter $D$. Wagner $e t a l$. averaged their results over 200 statistically independent velocity fields, covering over 800 dimensionless time units. Comparing this to LLO and EGG, that are averaged over 41 and 46 velocity fields, respectively, covering 40 and 60 dimensionless time units, respectively, it can be stated that the statistical error is expected to be much smaller for the VKW and WAG codes. This is visible in the skewness and flatness results presented later in this section.

To test the performance of the 3D PTV system, the wellknown mean axial velocity profile for turbulent pipe flow has been determined for $\operatorname{Re}_{b}=5300$ and 10300 and compared with corresponding results of EGG, VKW, and WAG. Results are shown in Fig. 5. Due to their negligible size, no error bars could be plotted. For both Reynolds numbers, two different DNS results are plotted but they are indistinguishable. No discrepancies between experimental and numerical results are found, implying that the flow is indeed a fully developed turbulent pipe flow. Experimental data are avail-
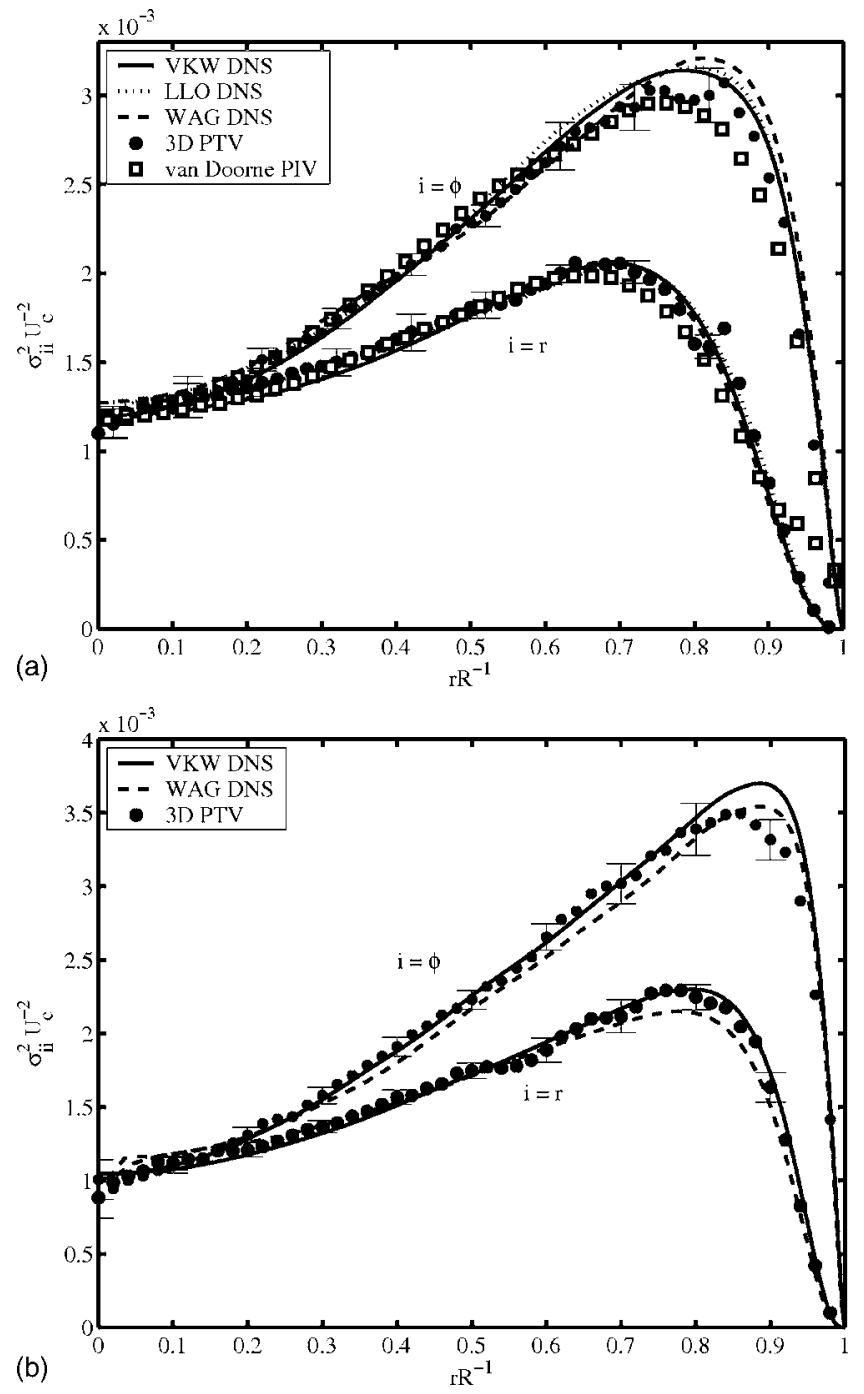

FIG. 6. Velocity MSV as a function of the dimensionless radius for radial and tangential velocity components at $\operatorname{Re}_{b}=5300$ (a) and $\operatorname{Re}_{b}=10300$ (b).

able up to $r / R \approx 0.97$. Even closer to the wall, light deflections hinder the measurements and no particles are detected here. The axial profile for the higher Reynolds number is also plotted normalized with the centerline velocity of the lower Reynolds number with a dotted line, showing the wellknown fact that the profile is flatter for higher Reynolds numbers.

The diagonal components of the Reynolds stress tensor $\sigma_{i j}=\left\langle u_{i}^{\prime} u_{j}^{\prime}\right\rangle$ are compared with VKW, LLO, and WAG DNS results for $\mathrm{Re}_{b}=5300$ and 10300 in Figs. 6 and 7. Furthermore, experimental results as obtained by van Doorne and Westerweel $^{30}$ and results from LDA measurements as performed in our laboratory are also included in the plots. These results are only available at the lower of the two Reynolds numbers, and the LDA results are only available for the axial diagonal component at $\operatorname{Re}_{b}=5300$. As we are interested in the non-normalized velocity correlation functions, it is important that the mean-square value (MSV) of the velocity is accurately determined. For all MSV plots, the 3D PTV data coincide with the DNS and LDA results within measurement error, even close to the wall. The only striking distinction is 


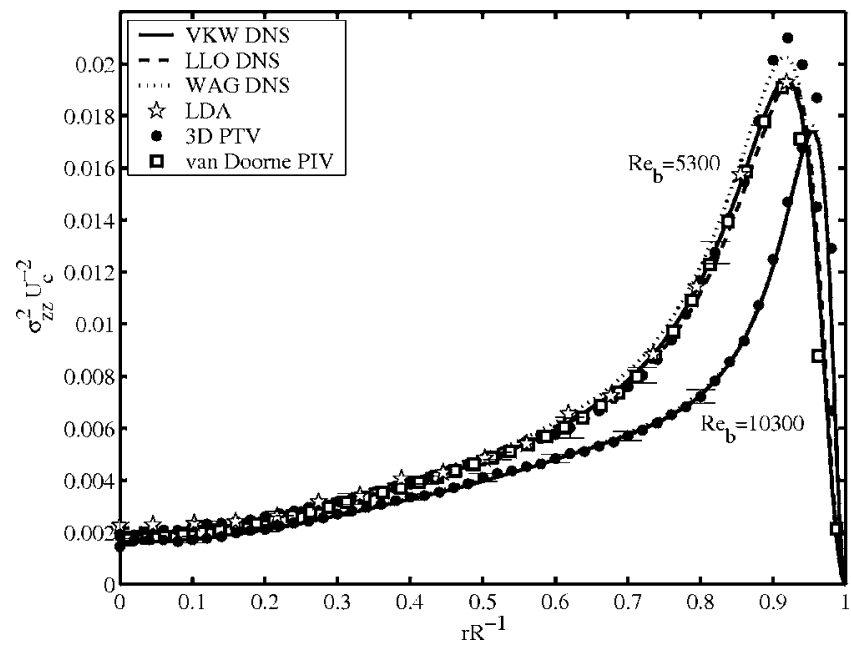

FIG. 7. Velocity MSV as a function of the dimensionless radius for axial velocity components at $\mathrm{Re}_{b}=5300$ and $\mathrm{Re}_{b}=10300$.

the lower 3D PTV $\sigma_{\phi \phi}^{2}$ values at radial positions around the peak in the profile. No other explanation than measurement inaccuracy can be given. The differences between the various DNS results are negligible. The agreement between the 3D PTV results and the PIV results of van Doorne and Westerweel is good, although deviations occur for $r / R>0.8$ for $\sigma_{\phi \phi}^{2}$ and close to the wall for $\sigma_{r r}^{2}$.

For isotropic flows, the nondiagonal terms of $\sigma_{i j}$ are all zero. But in the case of inhomogeneous turbulent flow, such as pipe flow, the only decoupled direction is the tangential one, which means that correlations like, e.g., $u_{\phi}^{\prime} u_{z}^{\prime}$ and $u_{\phi}^{\prime} u_{r}^{\prime}$, are zero. The only nonzero cross-component of $\sigma_{i j}$, the correlation of $u_{r}^{\prime}$ and $u_{z}^{\prime},\left\langle u_{r}^{\prime} u_{z}^{\prime}\right\rangle$, as calculated by VKW, LLO, and WAG, is compared with the current 3D PTV results and van Doorne and Westerweel ${ }^{30}$ PIV results in Fig. 8.

The results for all components of $\sigma_{i j}$ have also been compared with EGG DNS results. The results of Eggels et $a l .{ }^{26}$ showed significant deviations, especially close to the wall, from the presented DNS and experimental results and

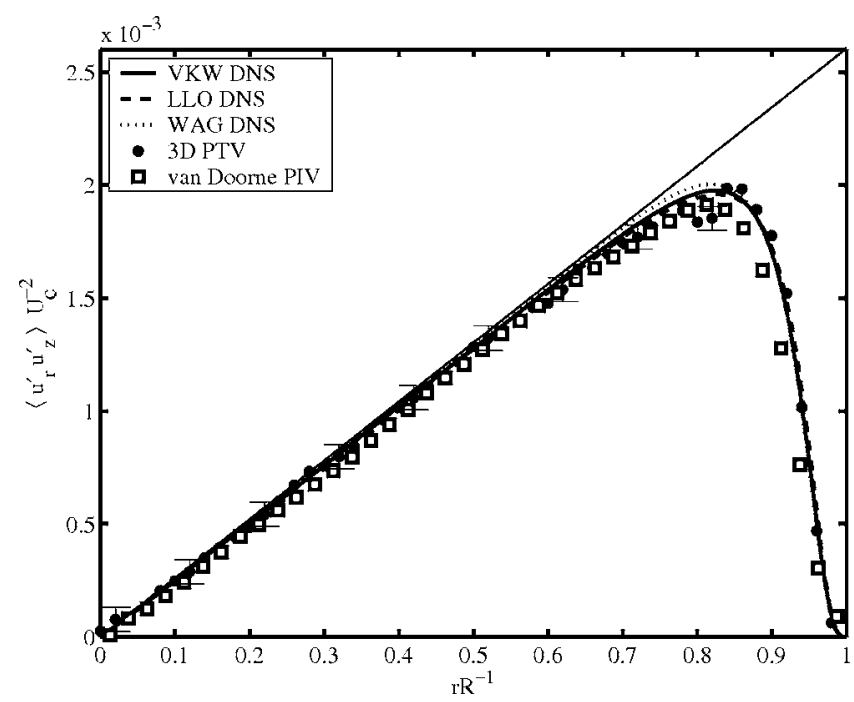

FIG. 8. Reynolds stress component $\left\langle u_{r}^{\prime} u_{z}^{\prime}\right\rangle$ as a function of the dimensionless radius at $\operatorname{Re}_{b}=5300$.
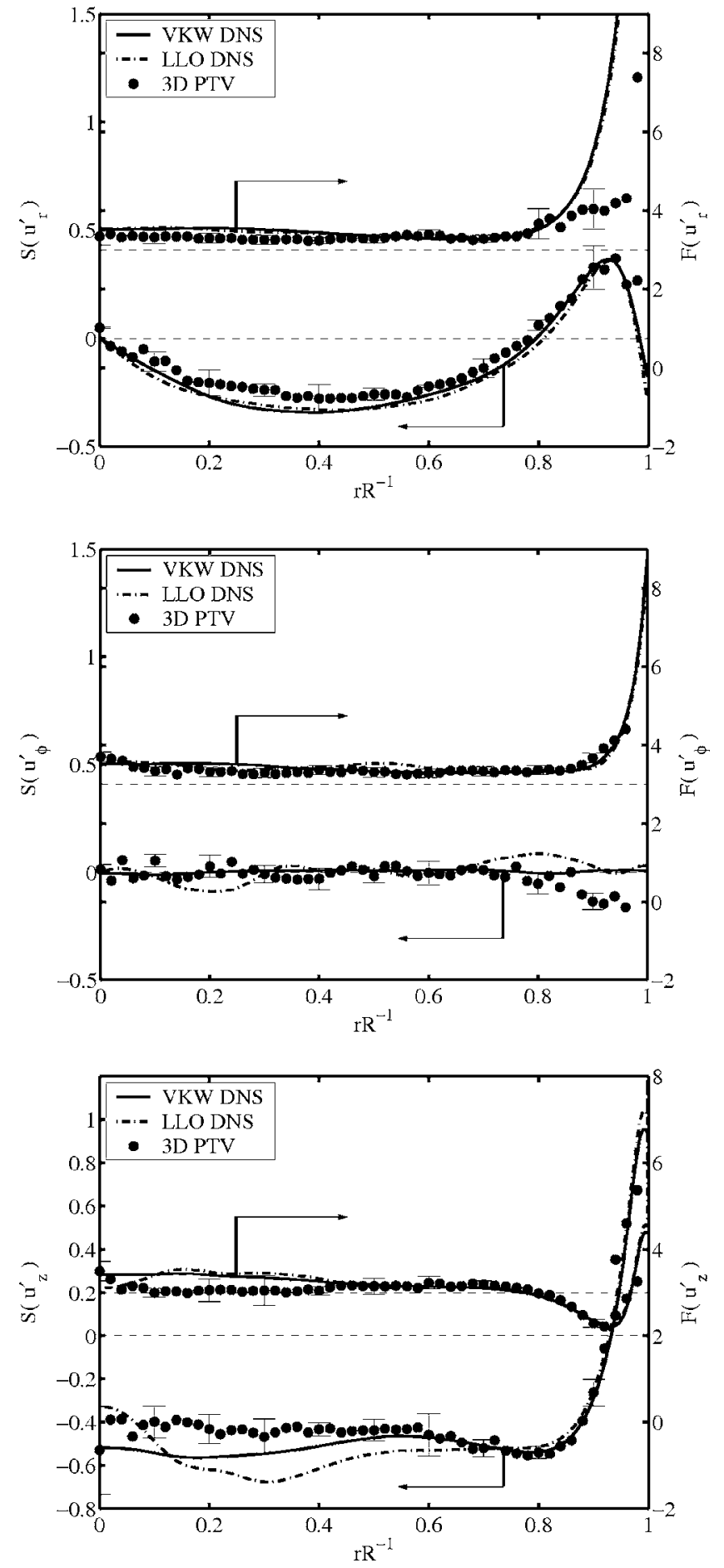

FIG. 9. Velocity skewness and flatness for all cylindrical velocity components at $\operatorname{Re}_{b}=5300$. The circles represent the 3D PTV results, the solid lines the VKW DNS, and the dash-dotted lines represent the LLO DNS.

have therefore been omitted from the figures. We believe that the deviations are caused by the coarser near-wall resolution used by Eggels et al.

The skewness factor $S=\left\langle u^{\prime 3}\right\rangle / \sigma^{3}$ and kurtosis or flatness factor $F=\left\langle u^{\prime 4}\right\rangle / \sigma^{4}$ of all velocity components are also determined and plotted in Fig. 9. These third- and fourth-order moments of the velocity are expected to be more sensitive to measurement noise as the lower-order moments presented above, but they show nevertheless a good agreement with the 
VKW and LLO DNS results. For inhomogeneous wallbounded flows, such as pipe flow, the PDFs of the velocity components are bell-shaped but not exactly Gaussian near the center. ${ }^{16,31}$ The departure from Gaussian behavior increases with decreasing distance to the wall as indicated by, for example, the data on turbulent channel flow by Moser $e t$ $a l .{ }^{31}$ This trend is also observed in Fig. 9 of the skewness and flatness factors, which are equal to $S=0$ and $F=3$ for Gaussian processes, respectively. The Gaussian values for the skewness and flatness are indicated by the thin dashed lines. The only significant deviation between 3D PTV and DNS is the failure of the 3D PTV results to capture the sudden increase in radial velocity flatness close to the wall for $F\left(u_{r}^{\prime}\right)$. This relatively large deviation can be explained by the cumulative effect of the relative measurement errors in $\left\langle u_{r}^{\prime 4}\right\rangle$ and $\sigma$. Although the absolute values of the error in both the second- and fourth-order moment of the radial velocity component are very small close to the wall, the relative error in the fourth order is large and this leads to a large relative error in the flatness as well. The large relative error in the fourthorder moment can be explained by the small amount of measurement data close to the wall. The departure from Gaussian behavior in the near-wall region is most obvious when observing the flatness profiles of the velocity components.

\section{LAGRANGIAN STATISTICS}

As mentioned in Sec. II, the unknown damping function in the Langevin equation can be determined from nonnormalized Lagrangian velocity correlation functions. For this procedure, the autocorrelation functions of all velocity components, as well as the cross-correlations, need to be known. These correlation functions are to be determined in the inertial subrange, which is considered to be the range $\tau_{k} \ll \tau \ll \tau_{c}$, with $\tau_{c}$ the Lagrangian integral time scale defined as $\tau_{c}=\int_{0}^{\infty} \rho(\tau) d \tau$. A typical estimate of $\tau_{c}$ for pipe flow is given by $D / U_{b}$. In other words, for an accurate description of velocity correlation functions, we have to be able to capture small-scale velocity fluctuations down to the Kolmogorov scale, $\tau_{k}$, as defined in Sec. II B, as well as particlevelocity tracks up to time separations specified below. The inertial subrange increases with increasing Reynolds number. At the moderate Reynolds numbers studied in this paper, the difference between the Kolmogorov and integral time scales is still relatively small. However, our DNS calculations have shown that, in order to capture the full inertial subrange, we have to follow particles up to time separations, $\tau$, given by $\tau u_{\tau} R^{-1}=0.06$. This corresponds to $0.8 \mathrm{~s}$ in the current experimental situation. This number, 0.06 , is only slightly dependent on the Reynolds number. To capture particle tracks up to $0.8 \mathrm{~s}$ with the restriction of a measurement volume with an axial length of $120 \mathrm{~mm}$, see Sec. II B, the maximum allowable axial velocity is $150 \mathrm{~mm} / \mathrm{s}$, which corresponds to $\operatorname{Re}_{b}$ $=13000$. This reasoning assumes perfect particle tracking characteristics of the 3D PTV algorithm; the particle track time will be verified in the following subsections. For higher fluid velocities in the current flow geometry, rigorous changes in the setup are needed. A camera and lightning system that moves along with the mean flow such as the one described by Virant and Dracos ${ }^{7}$ could offer a solution. In this way, the measurement volume moves along the flow with $U_{b}$ and particles can easily be followed for longer times than with stationary cameras.

Section IV A describes the velocity correlation function results and the determination of the damping function. The Kolmogorov constant also appears in the Langevin equation and can be determined from velocity structure functions as discussed in Sec. IV B.

\section{A. Velocity correlations}

Figure 10 compares Lagrangian velocity autocorrelation functions for the radial, tangential, and axial velocity components at two radial positions for 3D PTV with corresponding ones of VKW DNS at $\operatorname{Re}_{b}=5300$. The statistical error in the 3D PTV results is indicated by the dashed lines; for the DNS results it is more or less independent of time separation, $\tau$, and indicated by a single error bar. The figure also shows Eulerian velocity autocorrelation functions for the VKW DNS. These Eulerian correlations are calculated in a moving frame of reference, with the frame moving with the local average axial velocity,

$\rho_{i j}^{E}\left(\tau, r_{0}\right)=\left\langle u_{i}^{\prime}\left(r_{0}, \phi_{0}, z_{0}, t_{0}\right) u_{j}^{\prime}\left(r_{0}, \phi_{0}, z_{0}+\tau\left\langle u_{z}\left(r_{0}\right)\right\rangle, t_{0}+\tau\right)\right\rangle$.

The deviations between numerical and experimental results observed in the MSV plots of Figs. 6 and 7 manifest themselves here as offsets in the correlation starting points, because the correlation functions shown here are made dimensionless by $U_{c}$, but are not normalized. Without the application of the sampling method discussed in Sec. II C and the polynomial smoothing filter, the experimentally determined correlation functions would suffer from significantly too high starting values and noise peaks for small $\tau$ values. In the results presented in Fig. 10, the shape of the measured autocorrelation functions closely resembles that of the DNS results, even if some offset at $\tau=0$ occurs.

When comparing the correlation functions calculated in an Eulerian moving frame with their Lagrangian counterparts, it is clear that the correlations decorrelate faster in a Lagrangian reference frame. This can be explained by the movement of the Eulerian frame, which is only in the axial direction. In a Lagrangian reference frame, a particle is followed through the flow, thus also to other radial positions with other statistical properties leading to the faster decorrelation. The highly inhomogeneous behavior of the flow close to the wall causes the autocorrelations to decay faster there than in the central region of the pipe. This behavior is illustrated in Fig. 11, which shows tangential autocorrelation functions for $r / R=0.3$ and 0.9 that have been normalized with the corresponding starting values, $\sigma_{\phi \phi}^{2}$, to highlight the decay rate.

Figure 12 shows radial, tangential, and axial velocity autocorrelation functions at $\operatorname{Re}_{b}=10300$, as obtained by 3D PTV and VKW DNS. The higher fluid velocity at $\mathrm{Re}_{b}$ $=10300$ makes it impossible to follow the seeding particles as long as at the lower Reynolds number due to the limited axial length of the measurement volume. The maximum time 

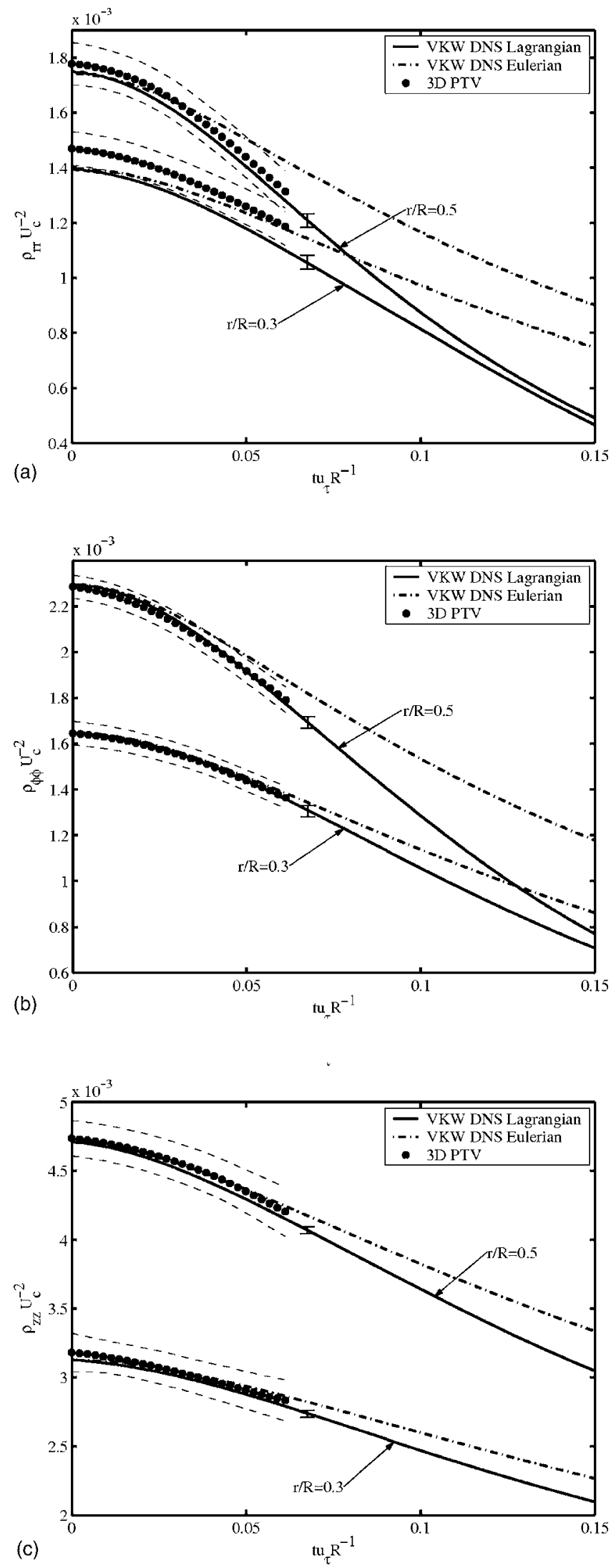

FIG. 10. Lagrangian and Eulerian velocity autocorrelation functions for the radial (a), tangential (b), and axial (c) velocity components at two different radial positions for VKW and 3D PTV at $\mathrm{Re}_{b}=5300$. The dashed lines indicate the statistical error for the 3D PTV results.

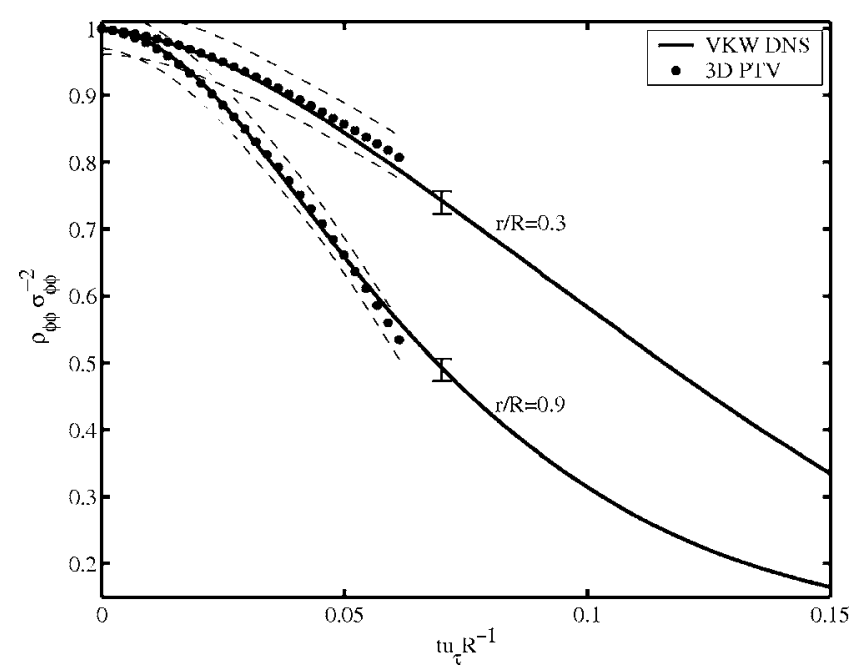

FIG. 11. Normalized Lagrangian velocity autocorrelation functions for the tangential velocity components at $r / R=0.3$ and 0.9 for VKW and 3D PTV at $\mathrm{Re}_{b}=5300$. The dashed lines indicate the statistical error for the 3D PTV results.

separation for which the correlation functions can be accurately determined thus decreases as compared to the lower Reynolds number. Furthermore, the dimensionless time resolution is not as good as at $\mathrm{Re}_{b}=5300$. Both effects can be observed when comparing Figs. 10 and 12. The Lagrangian experimental results are nevertheless in good agreement with the DNS results at this Reynolds number. However, the upper Reynolds number limit for the present setup is expected to be only slightly higher than 10300 . The upper limit estimate mentioned in Sec. IV of $\mathrm{Re}_{b}=13000$ therefore seems reasonable.

The coefficients of the damping tensor, $a_{i j}$, can be determined from the correlation functions in a way similar to that presented by Pope. ${ }^{3}$ The results for 3D PTV as well as DNS data are presented in Fig. 13, where the single error bar gives an indication of the statistical error. In a large part of the pipe, the coefficients are more or less constant; it is only near the wall that they strongly change. The region where the coefficients are more or less constant increases with increasing Reynolds number, but otherwise the dependence on the Reynolds number is small. The diagonal damping terms are all negative. The cross coefficient $a_{z r}$ is positive for all radial positions, increasing strongly in the wall region. The cross term $a_{r z}$ is close to zero for both Reynolds numbers over the whole radius. Some simplified Langevin models for inhomogeneous flows assume all nondiagonal damping terms to be zero. $^{32}$ These results show this assumption to be incorrect. Models without nondiagonal damping terms cannot produce correct results for both first- and second-order moments of particle velocity, ${ }^{33}$ as follows from application of the wellmixed criterion. The results in Fig. 13 show indeed that in inhomogeneous turbulence, $a_{z r}$ is not equal to zero and that the damping coefficients are position-dependent.

As discussed in Sec. III, there is no correlation between the tangential and the other velocity components. This implies that the only nonzero cross-correlation functions are $\rho_{r z}$ and $\rho_{z r}$, of which VKW DNS and 3D PTV results at two 

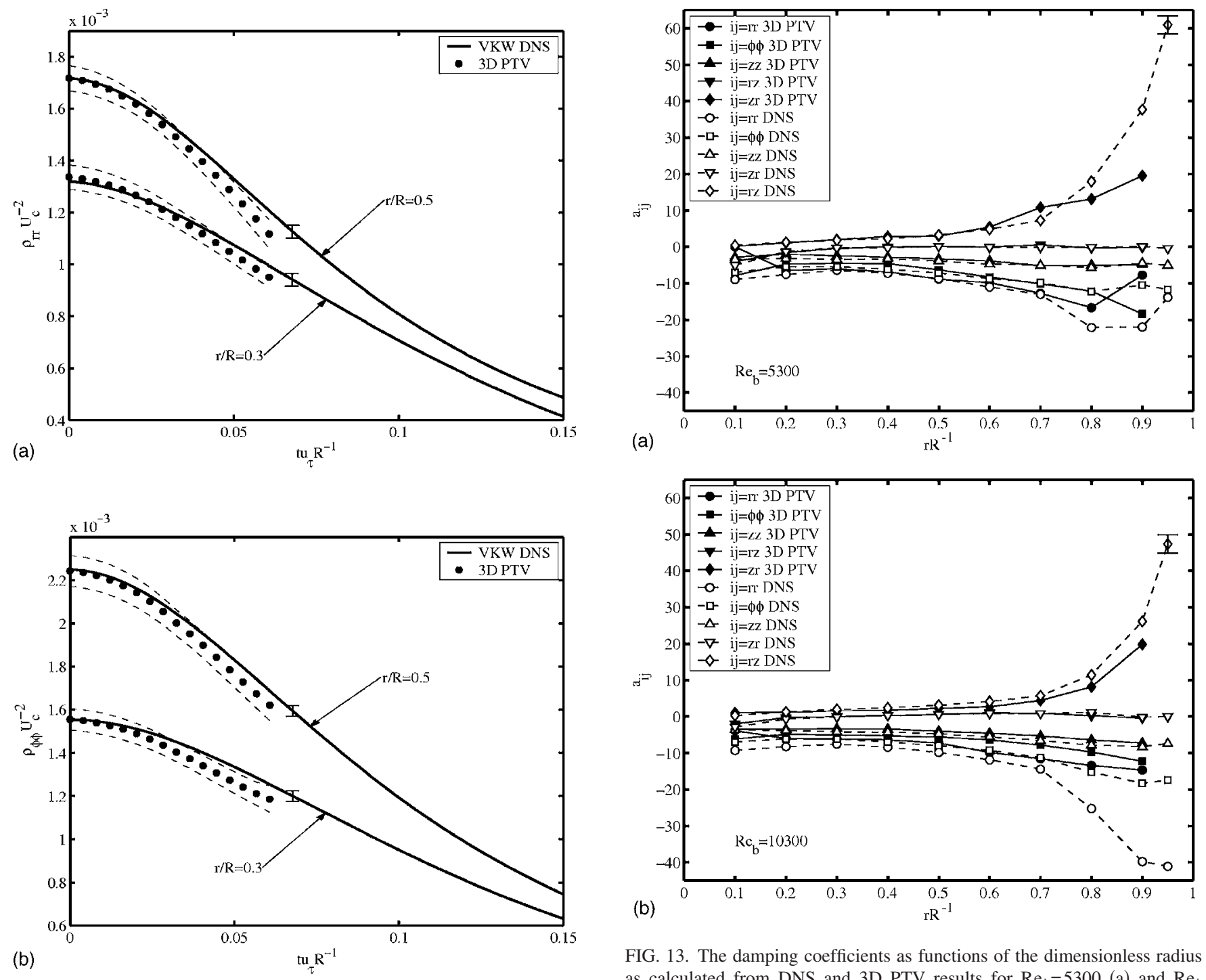

FIG. 13. The damping coefficients as functions of the dimensionless radius as calculated from DNS and 3D PTV results for $\mathrm{Re}_{b}=5300$ (a) and $\mathrm{Re}_{b}$ $=10300(\mathrm{~b})$.

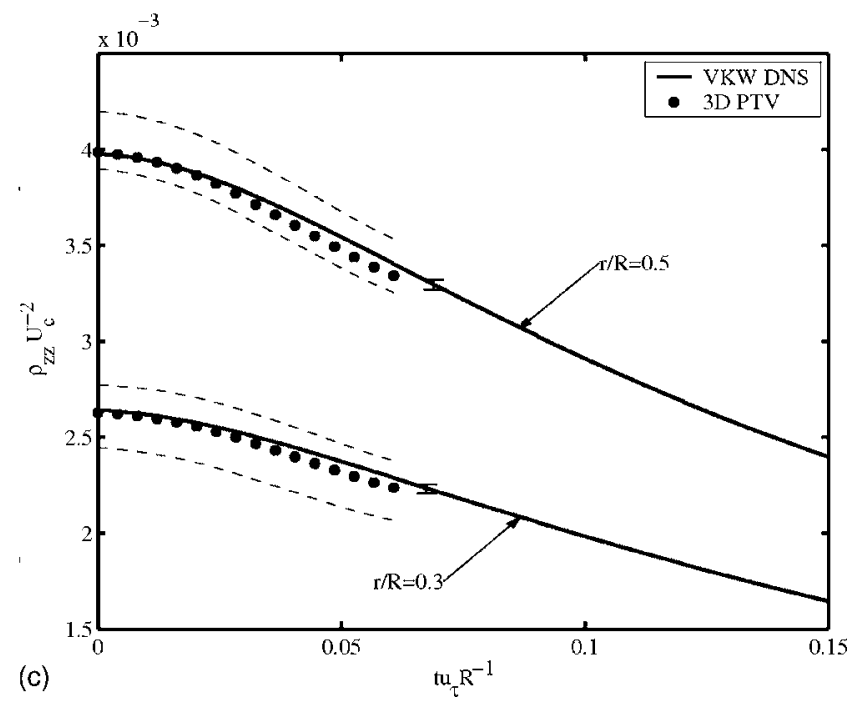

FIG. 12. Lagrangian velocity autocorrelation functions for the radial (a), tangential (b), and axial (c) velocity components at two different radial positions for VKW and 3D PTV at $\mathrm{Re}_{b}=10300$. The dashed lines indicate the statistical error for the 3D PTV results.

radial positions are shown in Fig. 14. As expected from the graph showing the Reynolds stress results (Fig. 8), the starting values of the experimental and numerical crosscorrelation function agree well. The cross-correlation functions also agree within statistical accuracy for $\tau>0$. Again, similar results have been found at the other radial positions measured. A striking difference between $\rho_{r z}$ and $\rho_{z r}$ is the considerably faster decay of $\rho_{z r}$. This phenomenon can be understood if one imagines a particle moving from radial position $r_{A}$ at time $t_{0}$ to radial position $r_{B}$ at time $t_{0}+\tau$. Without loss of generality, we take inward motion, i.e., $r_{B}<r_{A}$, implicating that $u_{r}^{\prime}\left(t_{0}\right)<0$ and $\left\langle u_{z}\left(r_{B}\right)\right\rangle>\left\langle u_{z}\left(r_{A}\right)\right\rangle$. The average product of $u_{r}^{\prime}$ and $u_{z}^{\prime}$ is positive (Fig. 8), so the particle is most likely to have a negative $u_{z}^{\prime}\left(t_{0}\right)$ as well. As follows from the Taylor hypothesis, a fluid particle tends to retain its velocity for a while. As a consequence, $u_{z}\left(t_{0}+\tau\right) \approx u_{z}\left(t_{0}\right)$. Since the average axial velocity component at the particle position increases in time, this results in an average increase of the absolute value of the axial velocity fluctuation. This makes the average product $\left\langle u_{r}^{\prime}\left(t_{0}\right) u_{z}^{\prime}\left(t_{0}+\tau\right)\right\rangle$ larger or about as large as $\left\langle u_{r}^{\prime}\left(t_{0}\right) u_{z}^{\prime}\left(t_{0}\right)\right\rangle$, which is exactly the trend observed in Fig. 


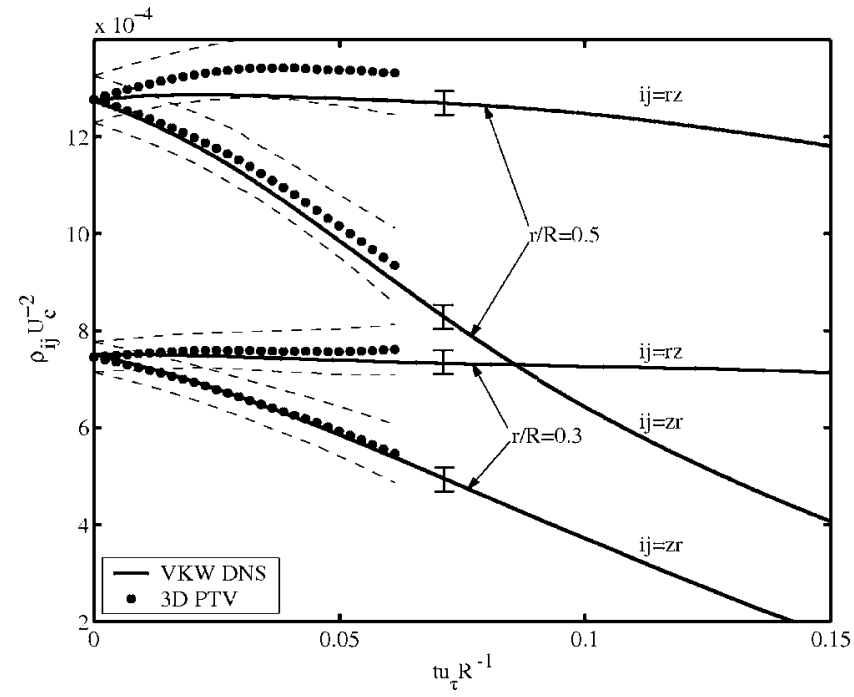

FIG. 14. Lagrangian velocity cross-correlation functions for VKW and 3D PTV at $\operatorname{Re}_{b}=5300$. The dashed lines indicate the statistical error for the 3D PTV results.

14. The argument is similar for a particle moving outwards. For $\left\langle u_{z}^{\prime}\left(t_{0}\right) u_{r}^{\prime}\left(t_{0}+\tau\right)\right\rangle$, this argument does not hold as $\left\langle u_{r}\right\rangle$ is constant and no average increase of the magnitude of the velocity fluctuation occurs.

The Taylor micro time scale, $\lambda$, is coupled to the second derivative of the velocity autocorrelation function as $\rho^{\prime \prime}(0)$ $=-2 / \lambda^{2}$, where the double prime denotes the second time derivative, see Tennekes and Lumley. ${ }^{34}$ The Taylor time scale is related to Lagrangian accelerations and it is a well-defined quantity that is often used in turbulent flow studies, among others, due to the clear relation with the Kolmogorov time scale, ${ }^{35} \lambda / u^{\prime}=\sqrt{15} \tau_{k}$. The Taylor time has been determined from DNS as well as 3D PTV results; see Fig. 15, which shows the variation of the direction-dependent $\lambda_{i}$ with radius and Reynolds number for each velocity component. The single error bar plotted gives an indication for the statistical error in all results. For the lower Reynolds number, $\lambda_{z}$ shows a strange behavior near the pipe wall. This might be due to a low Reynolds number effect or due to poor sampling, although it is consistent with the deviating behavior found for $C_{0}^{z^{*}}$, as presented later, in Sec. IV B. The Taylor time increases about linearly with increasing distance to the wall, which is to be expected because the near wall flow is dominated by the small viscous scales. With increasing Reynolds number, the influence of the viscous scales decreases, which might explain the decrease of both inhomogeneity and $\lambda$ values with increasing Reynolds number.

\section{B. Velocity structure functions}

Another important Lagrangian quantity is the secondorder Lagrangian velocity structure function, $D_{i i}(t)$, defined as

$$
D_{i i}(t)=\left\langle\left[v_{i}^{\prime}(t)-v_{i}^{\prime}(0)\right]^{2}\right\rangle .
$$

This quantity is important, since Kolmogorov's theory of local isotropy gives a scaling rule connecting the structure
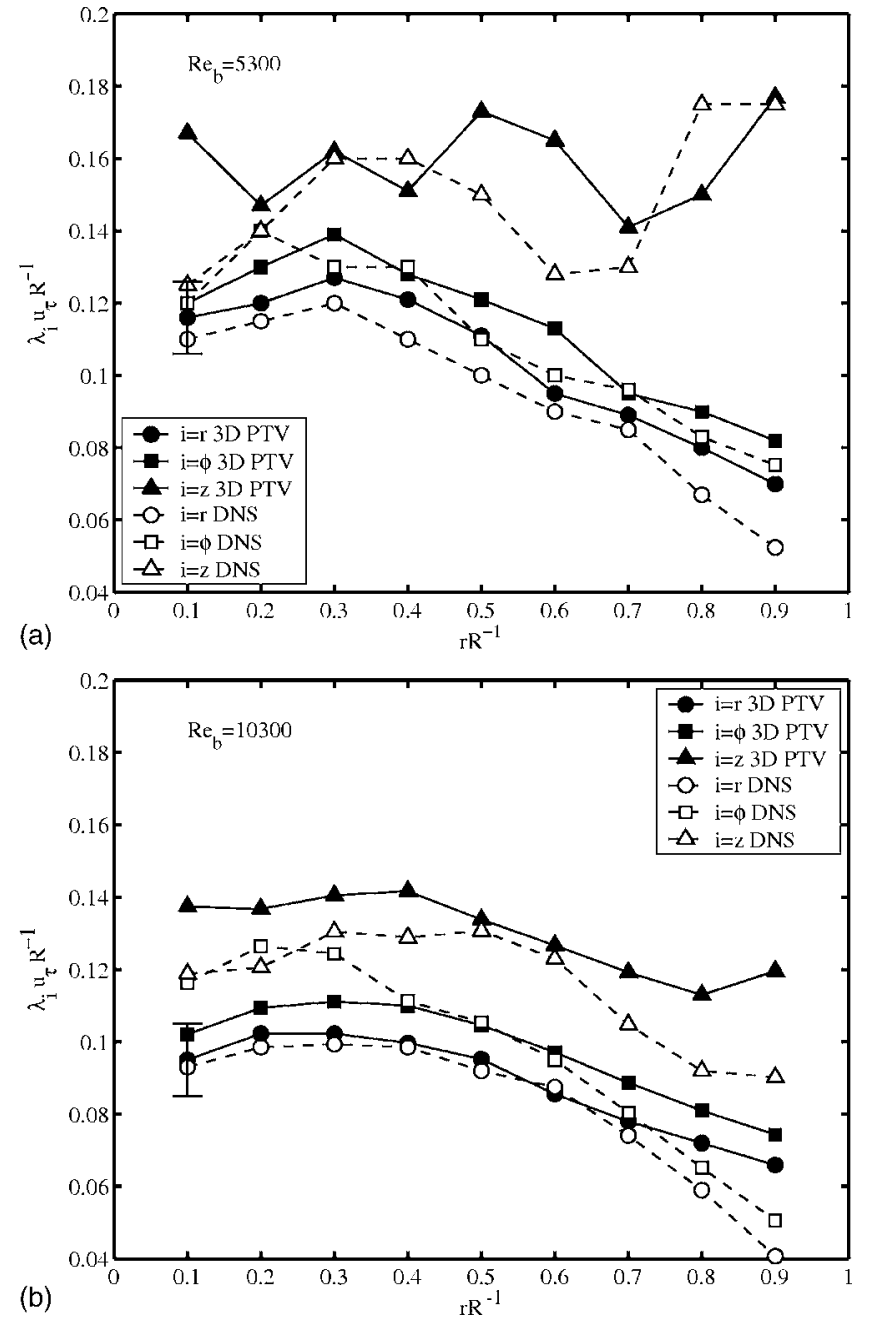

FIG. 15. The Taylor micro time scale as a function of the dimensionless radius as calculated from DNS and 3D PTV results for $\mathrm{Re}_{b}=5300$ (a) and $\mathrm{Re}_{b}=10300$ (b).

functions with the universal Kolmogorov constant, $C_{0}$; see, for example, Pope, ${ }^{35}$ p. 486 :

$$
D_{i i}(t)=C_{0}\langle\epsilon\rangle t .
$$

The scaling rule is valid in the inertial subrange, for time $t$ in the range $\tau_{k} \ll t \ll \tau_{c}$.

Finding the universal value for the Kolmogorov constant has been the subject of many studies over the past decades. In principle, $C_{0}$ can be determined from DNS computations, Lagrangian velocity measurements, as well as from tracer particle dispersion studies. An overview of some important results is given by Lien and D'Asaro. ${ }^{36}$ In the limit of very large Reynolds numbers, the structure functions in the three principal directions will be equal. However, this is not yet true at the Reynolds number studied here. It therefore stands to reason to define a direction-dependent $C_{0}^{i}$. This was also proposed by Pope $^{3}$ when he investigated a linear stochastic model for homogeneous shear flow. Lien and D'Asaro point out that the Taylor microscale Reynolds number, $R_{\lambda}$, must be greater than about $10^{5}$ for the inertial range of the structure function to be sufficiently wide to accurately determine $C_{0}$. According to Kolmogorov similarity, one should observe a 

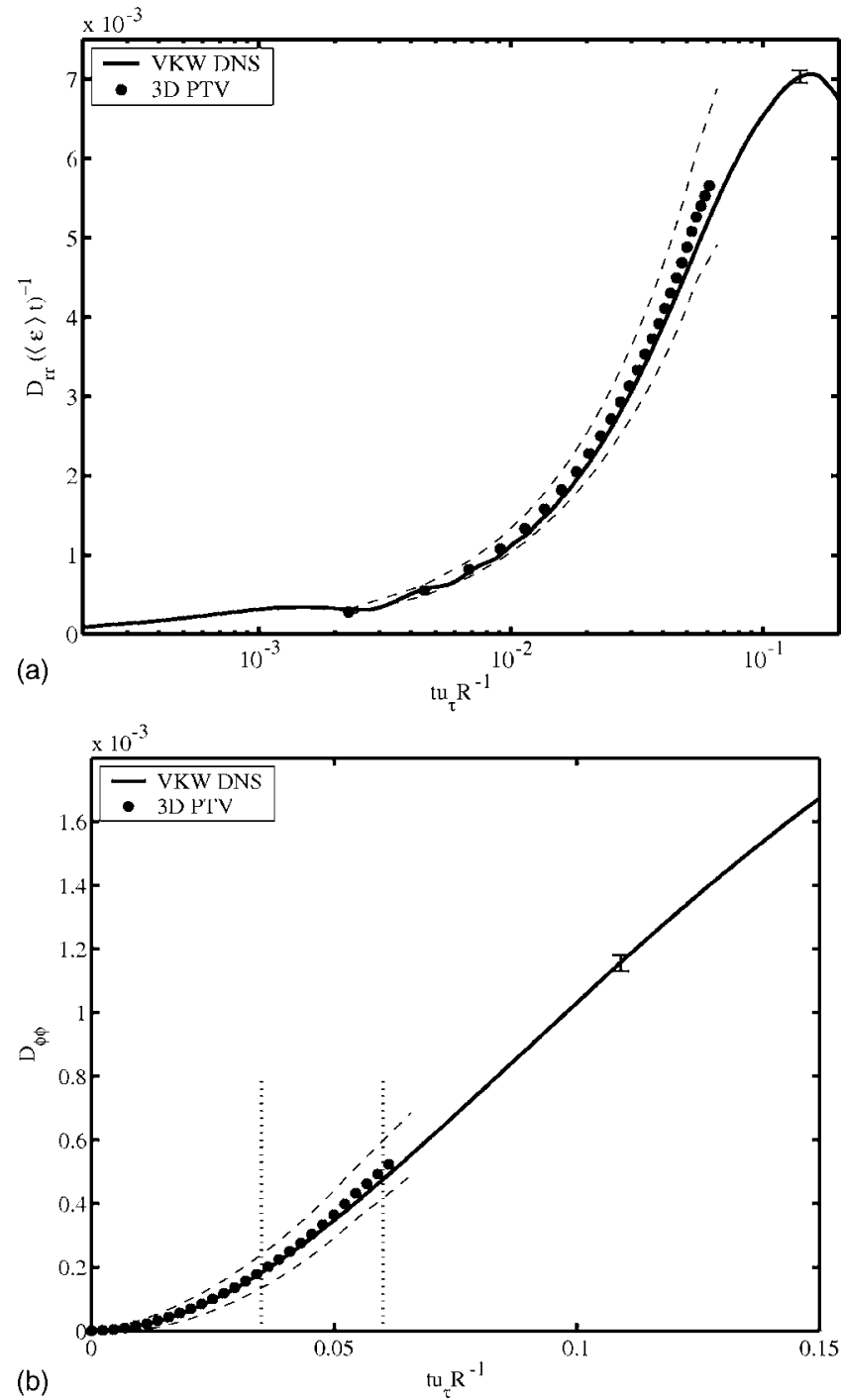

FIG. 16. Scaled Lagrangian velocity structure function for the radial velocity component (a) and unscaled structure function for the tangential velocity component (b) at $r / R=0.3$ at $\operatorname{Re}_{b}=5300$; VKW DNS and 3D PTV. The dashed lines indicate the statistical error for the 3D PTV results and the dotted lines in (b) indicate the inertial subrange.

plateau of $C_{0}$ in the inertial subrange. However, for the limited Reynolds numbers studied here, such plateaus are not present and a local maximum value, $C_{0}^{*}$, appears when the structure function is scaled with $\langle\epsilon\rangle t$. This local maximum is widely studied and is found to increase with $R_{\lambda} \cdot{ }^{36,37}$ Figure 16 shows radial and tangential structure functions for $r / R$ $=0.3$ at $\operatorname{Re}_{b}=5300$. For the DNS results, the structure functions are scaled as in Fig. 16(a) and the value of $C_{0}^{i *}$ is the local maximum. Due to the limited axial length of the measurement volume, 3D PTV results are not available up to the time separations where the local maximum occurs. For this case, the structure functions are not scaled, see Fig. 16(b), and fitted with a linear function in a time interval that is assumed to be in the inertial range, indicated with the dotted lines in the figure. Changing the time interval does not significantly alter the results for $C_{0}^{i^{*}}$ and the results obtained with this linear fit are similar to those obtained from DNS, as can be seen in Fig. 17, where the DNS and 3D PTV results
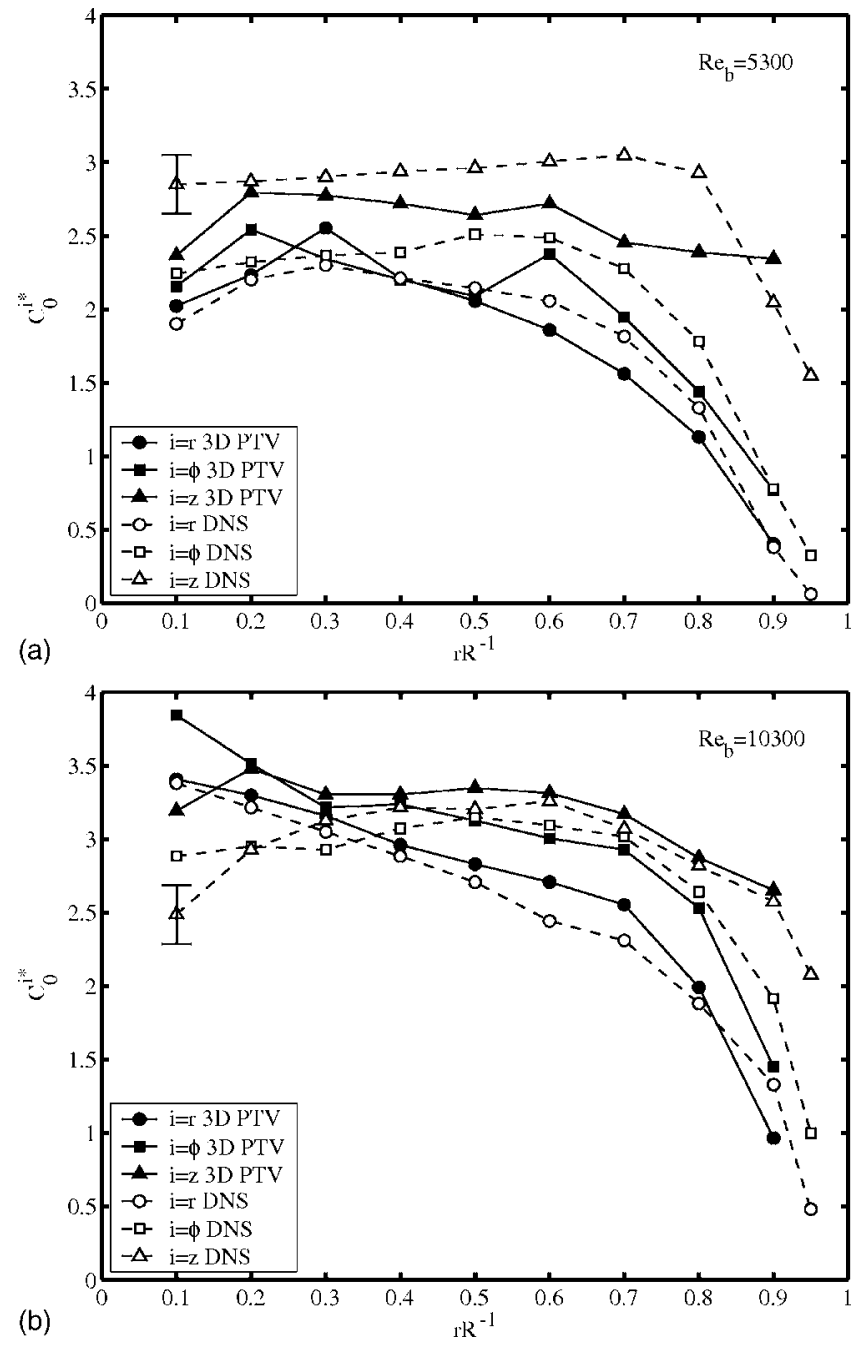

FIG. 17. The Kolmogorov constant as a function of the dimensionless radius as calculated from DNS and 3D PTV results for $\mathrm{Re}_{b}=5300$ (a) and $\mathrm{Re}_{b}$ $=10300(\mathrm{~b})$.

for $C_{0}^{i^{*}}$ are plotted. The single error bar plotted gives an indication for the statistical error in all results. In a large part of the pipe, $C_{0}^{i}$ has a constant value of approximately $2-2.5$ for $\operatorname{Re}_{b}=5300$ and around 3 for $\operatorname{Re}_{b}=10300$. The increase of $C_{0}^{i^{*}}$ with increasing distance to the wall is also found by Choi et $a l^{37}$ for turbulent channel flow. Furthermore, the anisotropy in the results decreases slightly with increasing Reynolds number.

When taking the limitations regarding $R_{\lambda}$ into account, the only high-quality independent measurements of $C_{0}$ $\operatorname{are}^{38,39} 5.5$ and 6.4. At the moment, the generally accepted idea is that $C_{0}$ increases with the Reynolds number and reaches an asymptotic value of approximately 6 to 7 . These findings are consistent with the relations proposed by Fox and Yeung: ${ }^{40}$

$$
C_{0}=6.5\left[1+\frac{8.1817}{R_{\lambda}}\left(1+\frac{110}{R_{\lambda}}\right)\right]^{-1} .
$$

The Taylor Reynolds number, $R_{\lambda}$, can be calculated ${ }^{35}$ using $R_{\lambda}=\sqrt{20 k^{2} /(3 \epsilon \nu)}$, where $k=\left\langle u_{i} u_{i}\right\rangle / 2$ is the turbulent kinetic energy. This relation gives $C_{0}=2.8$ and 3.7 for the turbulent 
Reynolds numbers studied here, $R_{\lambda}=30$ and 40 , which is in relatively good agreement with the presented results.

\section{CONCLUSION}

In the present study, a Lagrangian experimental technique, 3D PTV, has been applied to an inhomogeneous turbulent flow (pipe flow), which to our knowledge has not been done before. In addition, new DNS results for turbulent pipe flow obtained from a code with high spatial and temporal resolution are presented for the same Reynolds numbers that have been measured, $\operatorname{Re}_{b}=5300$ and 10300 . Some important aspects in processing raw 3D PTV data are discussed. For both Reynolds numbers and all three cylindrical velocity components, higher-order Eulerian velocity statistics, up to the fourth order, are studied. For $r / R \leq 0.97$, the agreement between DNS and 3D PTV results is found to be good.

Lagrangian statistics are studied through velocity correlation and structure functions. These quantities are used to determine the Kolmogorov constant $C_{0}$ and the damping coefficients in the Langevin model for fluid particle velocity. Good agreement between numerical and experimental results is found for both Reynolds numbers. Inhomogeneity, anisotropy, and main trends have been assessed and analyzed. The results show the necessity of incorporating nondiagonal damping terms in the Langevin model.

\section{ACKNOWLEDGMENTS}

This work was sponsored by the Stichting Nationale Computerfaciliteiten (National Computing Facilities Foundation, NCF) for the use of supercomputer facilities, with financial support from the Nederlandse Organisatie voor Wetenschappelijk Onderzoek (Netherlands Organization for Scientific Research, NWO).

${ }^{1}$ G. I. Taylor, "Diffusion by continuous movements," Proc. London Math. Soc. 20, 196 (1921).

${ }^{2}$ B. L. Sawford, "Turbulent relative dispersion," Annu. Rev. Fluid Mech. 33, 289 (2001).

${ }^{3}$ S. B. Pope, "Stochastic Lagrangian models of velocity in homogeneous turbulent shear flow," Phys. Fluids 14, 1696 (2002).

${ }^{4}$ N. Mordant, P. Metz, J.-F. Pinton, and O. Michel, "Acoustic technique for Lagrangian velocity measurement," Rev. Sci. Instrum. 76, 025105 (2005).

${ }^{5}$ N. Mordant, J. Delour, E. Léveque, A. Arnéodo, and J.-F. Pinton, "Long time correlations in Lagrangian dynamics: A key to intermittency in turbulence," Phys. Rev. Lett. 89, 254502 (2002).

${ }^{6} \mathrm{~B}$. Lüthi, A. Tsinober, and W. Kinzelbach, "Lagrangian measurement of vorticity dynamics in turbulent flow," J. Fluid Mech. 528, 87 (2005).

${ }^{7}$ M. Virant and T. Dracos, "3D PTV and its application on Lagrangian motion," Meas. Sci. Technol. 8, 1539 (1997).

${ }^{8} \mathrm{~J}$. Willneff, "A spatio-temporal matching algorithm for 3D particle tracking velocimetry," Ph.D. thesis, ETH Zurich (2003).

${ }^{9}$ R. N. Kieft, K. R. A. M. Schreel, G. A. J. van der Plas, and C. C. M. Rindt, "The application of a 3D PTV algorithm to a mixed convection flow," Exp. Fluids 33, 603 (2002).

${ }^{10}$ G. A. Voth, A. La Porta, A. M. Crawford, J. Alexander, and E. Bodenschatz, "Measurements of particle accelerations in fully developed turbulence," J. Fluid Mech. 469, 121 (2002).

${ }^{11} \mathrm{~S}$. Ott and J. Mann, "An experimental investigation of the relative diffusion of particle pairs in three-dimensional turbulent flow," J. Fluid Mech. 422, 207 (2000).

${ }^{12}$ K. Nishino, N. Kasagi, and M. Hirata, "Three-dimensional particle tracking velocimetry based on automated digital image processing," J. Fluids Eng. 111, 384 (1989).
${ }^{13}$ Y. Suzuki and N. Kasagi, "Turbulent air-flow measurement with the aid of 3-D particle tracking velocimetry in a curved square bend," Flow, Turbul. Combust. 63, 415 (2000).

${ }^{14}$ A. La Porta, G. A. Voth, A. M. Crawford, J. Alexander, and E. Bodenschatz, "Fluid particle accelerations in fully developed turbulence," Nature (London) 409, 1017 (2001).

${ }^{15}$ K. Hoyer, M. Holzner, B. Lüthi, M. Guala, A. Liberzon, and W. Kinzelbach, "3D scanning particle tracking velocimetry," Exp. Fluids 39, 923 (2005).

${ }^{16}$ M. P. B. Veenman, "Statistical analysis of turbulent pipe flow: A numerical approach," Ph.D. thesis, Technische Universiteit Eindhoven (2004).

${ }^{17}$ R. J. E. Walpot, P. C. J. N. Rosielle, and C. W. M. van der Geld, "Design of a set-up for high-accuracy 3D PTV measurements in turbulent pipe flow," Meas. Sci. Technol. 17, 3015 (2006).

${ }^{18}$ R. W. Miller, Flow Measurement Engineering Handbook, 3rd ed. (McGraw-Hill, New York, 1996).

${ }^{19}$ H. P. Bakewell and J. Lumley, "Viscous sublayer and adjacent wall region in turbulent pipe flow," Phys. Fluids 10, 1880 (1967).

${ }^{20}$ H. E. Albrecht, M. Borys, N. Damaschke, and C. Tropea, Laser Doppler and Phase Doppler Measurement Techniques (Springer-Verlag, New York, 2003).

${ }^{21}$ M. Marxen, P. E. Sullivan, M. R. Loewen, and B. Jühne, "Comparison of Gaussian particle center estimators and the achievable measurement density for particle tracking velocimetry," Exp. Fluids 29, 145 (2000).

${ }^{22}$ R. N. Kieft, "Mixed convection behind a heated cylinder," Ph.D. thesis, Technische Universiteit Eindhoven (2000).

${ }^{23}$ F. Yamamoto, T. Uemura, M. Iguchi, J. Ohta, A. Wada, and K. Mori, "3D PTV based on binary image correlations method and its applications to a mixing flow with a bubbling jet," in Computational Methods and Experimental Measurements, edited by C. A. Brebbia and G. M. Carlomagno (Springer, Berlin, 1993), Vol. VI, pp. 229-246.

${ }^{24}$ R. J. E. Walpot, J. G. M. Kuerten, and C. W. M. van der Geld, "Experimental determination of Lagrangian velocity statistics in turbulent pipe flow," Flow, Turbul. Combust. 76, 163 (2006).

${ }^{25}$ A. Savitzky and M. Golay, "Smoothing and differentiation of data by simplified least squares procedures," Anal. Chem. 36, 1627 (1964).

${ }^{26}$ J. G. M. Eggels, F. Unger, M. H. Weiss, J. Westerweel, R. J. Adrian, R. Friedrich, and F. T. M. Nieuwstadt, "Fully developed turbulent pipe flow: A comparison between direct numerical simulation and experiment," J. Fluid Mech. 268, 175 (1994).

${ }^{27}$ P. Loulou, "Direct numerical simulation of incompressible pipe flow using a b-spline spectral method," Ph.D. thesis, Stanford University (1996).

${ }^{28}$ C. Wagner, T. J. Hüttl, and R. Friedrich, "Low-Reynolds-number effects derived from direct numerical simulations of turbulent pipe flow," Comput. Fluids 30, 581 (2001).

${ }^{29}$ G. L. Squires, Practical Physics (Cambridge University Press, Cambridge, 2001).

${ }^{30}$ C. W. H. van Doorne and J. Westerweel, "Measurement of laminar, transitional and turbulent pipe flow using stereoscopic-piv," Exp. Fluids 42, 259 (2006).

${ }^{31}$ R. D. Moser, J. Kim, and N. N. Mansour, "Direct numerical simulation of turbulent channel flow up to $\operatorname{Re}_{\tau}=590$," Phys. Fluids 11, 943 (1999).

${ }^{32}$ I. Iliopoulis and T. J. Hanratty, "Turbulent dispersion in a nonhomogeneous field," J. Fluid Mech. 392, 45 (1999).

${ }^{33}$ D. J. Thomson, "Criteria for the selection of stochastic models of particle trajectories in turbulent flows," J. Fluid Mech. 180, 529 (1987).

${ }^{34}$ H. Tennekes and J. L. Lumley, A First Course in Turbulence (MIT, Cambridge, 1972).

${ }^{35}$ S. B. Pope, Turbulent Flows (Cambridge University Press, Cambridge, 2000).

${ }^{36}$ R. C. Lien and E. A. D'Asaro, "The Kolmogorov constant for the Lagrangian velocity spectrum and structure function," Phys. Fluids 14, 4456 (2002).

${ }^{37}$ J. I. Choi, Y. Kyongmin, and L. Changhoon, "Lagrangian statistics in turbulent channel flow," Phys. Fluids 16, 779 (2004).

${ }^{38}$ N. Mordant, P. Metz, O. Michel, and J. F. Pinton, "Measurements of Lagrangian velocity in fully developed turbulence," Phys. Rev. Lett. 87, 214501 (2001).

${ }^{39} \mathrm{P}$. K. Yeung, "Lagrangian characteristics of turbulence and scalar transport in direct numerical simulations," J. Fluid Mech. 427, 241 (2001).

${ }^{40}$ R. O. Fox and P. K. Yeung, "Improved Lagrangian mixing models for passive scalars in isotropic turbulence," Phys. Fluids 15, 961 (2003). 